\title{
Um Modelo para Criação de Jogos Sérios e Ubíquos orientados à Aprendizagem baseada em Problemas
}

\author{
Sandro O. Dorneles, Cristiano A. da Costa, Sandro J. Rigo. Jorge L. V. Barbosa \\ Programa de Pós-Graduacão em Computacão Aplicada - PPGCA \\ Universidade do Vale do Rio dos Sinos - UNISINOS \\ Av. Unisinos, 950 - Cristo Rei - Sao Leopoldo/RS - CEP: 93022-750 \\ pfsandro@yahoo.com.br, \{cac, rigo, jbarbosa $\}$ dunisinos.br
}

\begin{abstract}
The easy access and evolution of mobile devices enables new educational scenarios with challenges and integration opportunities. Different games have been developed in recent years to support teaching and learning in the most different areas. In this way, this article proposes a model for the development of serious and ubiquitous games oriented to problem-based learning. The model allows the teacher to create games and adapt to real-world scenarios. The evaluation of the model was conducted through a prototype tested with a group of 13 teachers from different areas that indicated satisfactory results and which indicated intention to use the model. A second evaluation involving 22 students from the 8th year of elementary school indicated that the game created allowed motivation and engagement.
\end{abstract}

Resumo. O fácil acesso e evolução dos dispositivos móveis, possibilita novos cenários educativos com desafios e oportunidades de integração. Diferentes jogos foram desenvolvidos nos últimos anos para apoiar o ensino e aprendizagem nas mais diferentes áreas. Dessa forma, esse artigo propõe um modelo para o desenvolvimento de jogos sérios e ubíquos orientados à aprendizagem baseada em problemas. O modelo permite ao professor a criação de jogos e a adaptação para cenários do mundo real. A avaliação do modelo foi conduzida através de um protótipo testado com um grupo de 13 professores de diferentes áreas que indicaram resultados satisfatórios e que apontam intenção de uso do modelo. Uma segunda avaliação envolvendo 22 alunos do $8^{\circ}$ ano do ensino fundamental indicou que o jogo criado permitiu motivação $e$ engajamento.

\section{Introdução}

A evolução das tecnologias e a integração de dispositivos móveis em atividades de aprendizagem têm atraído a atenção de educadores e pesquisadores [Khan et al. 2015], [Huang e Chiu 2015]. Os dispositivos móveis e a tecnologia de Internet sem fio possibilitam novas abordagens educacionais com diferentes e variados recursos tecnológicos em qualquer lugar e a qualquer momento [Cárdenas-Robledo e PeñaAyala 2018].

Com isso, ampliam-se as teorias e os métodos de aprendizagem que estimulam e motivam o aluno através do uso das tecnologias, e entre elas, destacam-se o uso de jogos educacionais com resultados significativos de aprendizagem [Hainey et al. 2016]. Prensky [2012] entende ainda que a utilização de jogos pode promover a aproximação entre os nativos digitais e os imigrantes digitais, permitindo assim ao professor e aluno alternarem os papéis na busca do conhecimento. 
VIII Congresso Brasileiro de Informática na Educação (CBIE 2019)

Anais do XXX Simpósio Brasileiro de Informática na Educação (SBIE 2019)

O uso de jogos para auxiliar no desenvolvimento cognitivo, não é uma novidade, a mudança está na possibilidade de aliar tecnologias que permitem a expansão de uma brincadeira, de uma diversão ou ainda competição, para além do círculo mágico definido por Huizinga [2008], que caracteriza o jogo como sendo um elemento definido pelo social, temporal e espacial. A possibilidade de um jogo ser contextualizado no mundo real e não apenas de forma virtual, não só como fator de diversão, mas também com objetivos educacionais bem definidos, podem apresentar novas oportunidades de interação entre o saber e o fazer.

A ampliação do jogo para o mundo real baseia-se nas possibilidades da computação ubíqua [Weiser 1991] que prevê a criação de ambientes saturados de computação e capacidade de comunicação integrando-os com usuários. Dessa forma, cresce também a possibilidade de associar e ampliar conteúdos de aprendizagem com situações reais do dia a dia.

Contudo, a mesma tecnologia que traz facilidades, impõe mudanças. A crescente facilidade com que os jovens se apropriaram desses recursos, exige um novo entendimento social, cultural e educacional. Em Santaella [2014], é proposto como alternativa o uso de recursos móveis e ubíquos, favorecendo dessa forma, processos de aprendizagem abertos, podendo ser compartilhado e resolvido de forma colaborativa, utilizando os recursos de conectividade individual e personalizada oferecidos pelos dispositivos móveis.

Esse artigo apresenta a avaliação de um modelo para construção de jogos sérios e ubíquos para apoio ao aprendizado baseado em problemas, denominado Uchallenge. O artigo também descreve um protótipo para a criação dos jogos pelo professor e sua aplicação usando dispositivos móveis por alunos.

Este trabalho está organizado da seguinte forma: a Seção 2 apresenta os trabalhos relacionados com o tema da pesquisa; na Seção 3 é apresentado o modelo Uchallenge e suas características; a Seção 4 apresenta a avaliação e os resultados obtidos, e a seção 5, apresenta as conclusões finais e trabalhos futuros.

\section{Trabalhos relacionados}

Essa seção examina trabalhos realizados por outros pesquisadores, que apresentam contribuições no mesmo foco deste estudo, identificando possibilidades de ampliação nessa área. Assim, destacam-se relatos de jogos desenvolvidos com características ubíquas que buscam aproximar a tecnologia do real e potencializar a aprendizagem.

Laine et al. [2010] relatam estudos realizados num período de três anos pela equipe da Ubiquelab da Universidade Eastern, Finlândia. As experiências são contadas com base no desenvolvimento de jogos ubíquos utilizando um modelo de design hipercontextualizado, ou seja, "onde o jogo está enraizado no mesmo contexto em que o jogador está incorporado".

Weatherlings é um jogo de estratégia de cartas colecionáveis, semelhante a vários outros jogos Trading Card Games (TCG). Segundo Klopfer et al. [2012], o jogo tem como objetivo, proporcionar que os usuários "sejam capazes de ler com sucesso mesas climáticas para planejar estratégias apropriadas para dados climáticos históricos reais". 
VIII Congresso Brasileiro de Informática na Educação (CBIE 2019)

Anais do XXX Simpósio Brasileiro de Informática na Educação (SBIE 2019)

Hwang et al. [2016] apresentam um jogo baseado em Realidade Aumentada (RA) como forma de contextualizar um ambiente real. O jogo ocorre em um jardim de borboletas e plantas hospedeiras. Para interação com o ambiente real são usados redes de comunicação sem fio, tags de QRcode e sistemas de localização.

Tais experiências apresentam relatos do desenvolvimento de jogos em áreas e contextos específicos de aprendizagem não sendo possível adaptá-los para diferentes ambientes e objetivos. Dessa forma na busca de facilitar a construção de jogos em diferentes áreas do conhecimento foi desenvolvido o modelo Uchallenge com $\mathrm{o}$ propósito de criar uma plataforma que permita a construção dos jogos ubíquos pelo professor em diferentes áreas e ambientes de aprendizagem.

\section{Modelo Uchallenge}

O modelo proposto fornece uma plataforma para criação de jogos sérios e ubíquos, apoiado em uma metodologia de aprendizagem baseada em problemas. Na proposta, o professor pode gerenciar o jogo, definindo o domínio de aprendizagem a ser utilizado, os conteúdos, problemas e desafios que serão usados em cada etapa da aprendizagem e do jogo, bem como, quais recursos ubíquos podem ser explorados no ambiente em que o jogo se realizará. O modelo Uchallenge é apresentado na Figura 1, dividido em dois módulos: módulo professor, responsável pela criação do jogo, e o módulo aluno, aplicação móvel gerada a partir da criação do jogo pelo professor.

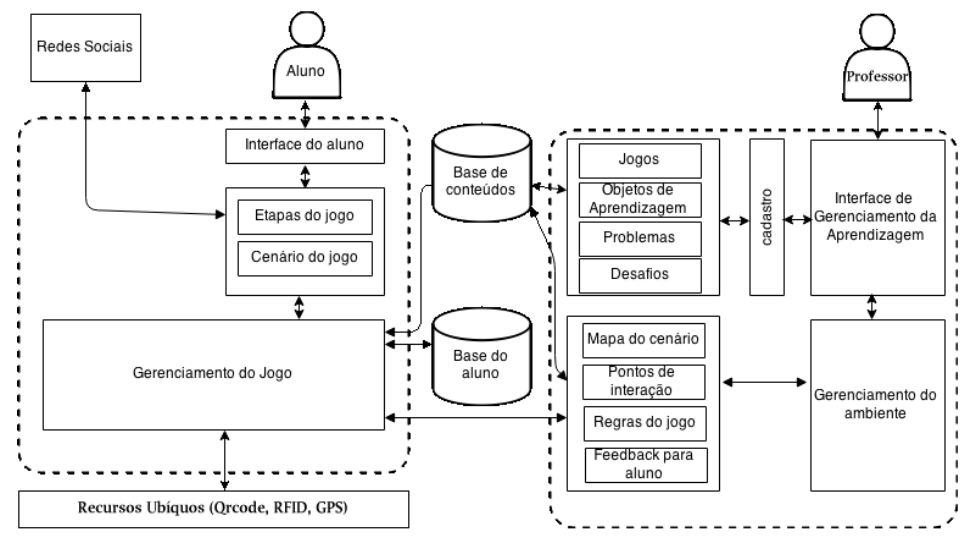

Figura 1: Modelo Uchallenge

O módulo do professor permite autenticação, gerenciamento de usuários, criação e manutenção do jogo, inserção e manutenção de objetos de aprendizagem, criação e gerenciamento de ações, associação de interações ao ambiente e geração de sensores para ambiente. O módulo aluno possibilita o acesso por meio de uma aplicação móvel, sendo possível autenticar usuário, acessar jogos disponíveis e recursos de ambiente e executar todas as etapas do jogo.

\section{Avaliação e Resultados}

A fim de comprovar de maneira sistemática os resultados da utilização do Uchallenge para criação de jogos ubíquos em diferentes áreas de aprendizagem e a motivação proporcionada aos jogadores, foram coletadas evidências que permitissem analisar e comprovar as seguintes hipóteses de pesquisa: 
VIII Congresso Brasileiro de Informática na Educação (CBIE 2019)

Anais do XXX Simpósio Brasileiro de Informática na Educação (SBIE 2019)

- o uso do modelo Uchallenge favorece a criação e uso de jogos sérios para aprendizagem baseada em problemas em diferentes áreas de aprendizagem;

- o uso de jogos criados a partir do Uchallenge proporcionam motivação e engajamento durante o jogo.

Os procedimentos de avaliação do modelo foram divididos em duas etapas. A avaliação com professores de diferentes áreas de aprendizagem, com a finalidade de verificar a percepção em relação a utilidade e facilidade de uso na criação dos jogos e a avaliação com alunos, a fim de mensurar o nível de motivação e engajamento durante o jogo.

\subsection{Avaliação por professores}

O Technology Acceptance Model (TAM) tem sido aplicado na avaliação da aceitação de softwares com objetivos educacionais [Persico et al. 2014]. A metodologia de avaliação proposta para o Uchallenge usou além do modelo TAM adaptado, variáveis sugeridas no modelo de Prieto et al. [2014], tais como, experiência anterior, diversão e condições facilitadoras.

A seleção dos itens para o questionário considerou variáveis externas (VE) de experiências do professor com o lúdico e área de atuação, para analisar dois aspectos: percepção de utilidade (PU) e percepção de facilidade de uso (PFU), baseados em [Wallace e Sheetz 2014]. O questionário é apresentado na Tabela 1.

Tabela 1: Detalhamento do questionário

\begin{tabular}{|l|l|}
\hline \multicolumn{2}{|l|}{ Variáveis (VE) } \\
\hline VE1 & Área de atuação/disciplina. \\
\hline VE2 & Costuma utilizar elementos lúdicos em suas aulas? \\
\hline VE3 & Utiliza tecnologias como ferramentas de apoio? \\
\hline VE4 & Usa algum tipo de jogo para sua diversão frequentemente? \\
\hline \multicolumn{2}{|c|}{ Percepção de utilidade (PU) } \\
\hline PU1 & Sua área de conhecimento permite integração entre teoria e prática. \\
\hline PU2 & Jogos educativos podem motivar a aprendizagem em sua área de conhecimento. \\
\hline PU3 & O modelo Uchallenge permite explorar ambientes reais da aprendizagem. \\
\hline PU4 & O modelo Uchallenge facilita o desenvolvimento de autonomia do aluno. \\
\hline PU5 & Eu usaria jogos criados no Uchallenge com meus alunos. \\
\hline \multicolumn{2}{|c|}{ Percepção de facilidade de uso (PFU) } \\
\hline PFU1 & A interface do Uchallenge é de fácil compreensão. \\
\hline PFU2 & $\begin{array}{c}\text { As informações sobre a criação do jogo e recursos disponíveis são apresentadas de forma } \\
\text { clara e objetiva. }\end{array}$ \\
\hline PFU3 & A criação das ações como perguntas, desafios e quiz são de fácil entendimento. \\
\hline PFU4 & $\begin{array}{c}\text { A definição do mapa do cenário e associação de eventos a esse cenário é de fácil } \\
\text { compreensão. }\end{array}$ \\
\hline PFU5 & Tive facilidade em todas as etapas de criação do jogo. \\
\hline
\end{tabular}

Os resultados obtidos na pesquisa permitiram avaliar as seguintes hipóteses:

- H0: As variáveis externas (VE) de experiência influenciam na decisão de uso do modelo;

- H1: A percepção de facilidade de uso (PFU) tem impacto na percepção de utilidade (PU);

- H2: O modelo Uchallenge pode ser adotado por diferentes áreas de aprendizagem. 
VIII Congresso Brasileiro de Informática na Educação (CBIE 2019)

Anais do XXX Simpósio Brasileiro de Informática na Educação (SBIE 2019)

O estudo foi realizado com 13 docentes de escolas da Rede Pública e Privada do estado. A pesquisa foi realizada individualmente com os professores, onde foi apresentado o Uchallenge, seus objetivos e etapas para construção de um jogo. Em seguida, uso do modelo e construção de uma sessão de jogo, que implicava em definir o tema, nome e cenário onde o jogo seria ambientado, problemas, desafios e conteúdos que seriam propostos para o aluno, além de definir pontos de interação para os desafios e sensores para o ambiente. Após a sessão de uso do Uchallenge, o professor foi convidado a responder o questionário para coleta de dados.

Os resultados obtidos na análise de variáveis externas, permitiram identificar o perfil do professor quanto a sua área de atuação e tendência ao uso de jogos e tecnologias. Dessa forma, os resultados distinguem um número de oito áreas contempladas na avaliação. Dessas 30,8\% são de línguas: Português, Alemão e Inglês; 15,4\% Matemática e Administração; 7,7\% Química, História, Geografia, Ciências e Informática.

Os resultados apontam também que $54 \%$ dos professores utilizam o lúdico como forma de apoio em sala de aula frequentemente; $38 \%$ às vezes, e somente $8 \%$ afirmam não utilizar esse tipo de recurso. Pode-se verificar ainda, que $85 \%$ utilizam a tecnologia frequentemente e $15 \%$, às vezes. Quanto ao uso de jogos para diversão pessoal, $31 \%$ dizem não fazer uso desse tipo de entretenimento, enquanto que $69 \%$ dizem fazer uso de jogos.

As informações iniciais do perfil do professor indicam que a maioria independentemente da área de atuação, utilizam jogos e tecnologias em suas práticas, e mais de $65 \%$ gostam e fazem uso de jogos no dia a dia. Quanto a percepção de utilidade, levou-se em consideração para análise, a integração entre teoria e prática de cada área de avaliação e as possibilidades de uso de um jogo que permitisse essa integração, além de explorar ambientes reais e a perspectiva de que o jogo possa motivar e estimular a autonomia do aluno.

A Tabela 2 apresenta os resultados da percepção de utilidade do modelo. Para melhor descrição dos resultados os itens 1 - discordo totalmente e 2 - discordo, foram unidos, assim como os itens 4 - concordo e 5 - concordo totalmente, enquanto que, o item 3 foi considerado neutro.

Tabela 2. Resultados Constructor Percepção de Utilidade

\begin{tabular}{|l|l|l|l|l|l|}
\hline & RM & Mediana & Não concordo (1-2) & Neutro (3) & Concordo (4-5) \\
\hline PU1 & 4,31 & 4 & $0-0 \%$ & $2-15 \%$ & $11-85 \%$ \\
\hline PU2 & 4,92 & 5 & $0-0 \%$ & $0-0 \%$ & $13-100 \%$ \\
\hline PU3 & 4,92 & 5 & $0-0 \%$ & $0-0 \%$ & $13-100 \%$ \\
\hline PU4 & 4,77 & 5 & $0-0 \%$ & $0-0 \%$ & $13-100 \%$ \\
\hline PU5 & 5 & 5 & $0-0 \%$ & $0-0 \%$ & $13-100 \%$ \\
\hline
\end{tabular}

A Tabela 2 apresenta o Ranking Médio (RM) e a mediana. O RM é calculado pela média ponderada (MP) dividida pelo $\mathrm{n}^{\mathrm{o}}$ de respostas (NR), RM=MP/NR, dessa forma quanto mais próximo de $5 \mathrm{o}$ RM estiver, maior será o nível de satisfação dos respondentes e quanto mais próximo de 1 menor a satisfação.

A análise individual dos valores de Ranking Médio, Mediana e da porcentagem das variáveis do constructor de percepção de utilidade apontam resultados 
VIII Congresso Brasileiro de Informática na Educação (CBIE 2019)

Anais do XXX Simpósio Brasileiro de Informática na Educação (SBIE 2019)

significativos. A maioria dos respondentes conseguem relacionar em sua área de atuação teoria e prática (PU1 85\%), e percebem os jogos educativos como uma ferramenta capaz de motivar a aprendizagem nessas áreas (PU2 100\%). É possível considerar ainda, que o Uchallenge permite explorar ambientes de aprendizagem reais (PU3 100\%), e que pode contribuir para o desenvolvimento de autonomia do aluno (PU4 100\%). A soma desses resultados pode ser percebida na variável PU5, onde 100\% dos respondentes indicam que usariam o Uchallenge com seus alunos.

O mesmo processo de análise foi realizado no constructor percepção de facilidade de uso na Tabela 3. De acordo com os resultados percebe-se que $100 \%$ dos respondentes consideram a interface de fácil compreensão (PFU1), que as informações para criação do jogo são claras (PFU2 92\%), que a criação de ações é de fácil entendimento (PFU3 85\%), e que a definição de mapas e associação a eventos é de fácil compreensão (PFU 100\%). No entanto, apesar dos resultados das demais variáveis serem satisfatórios, $23 \%$ dos professores indicaram algum tipo de dificuldade em relação a criação dos jogos.

Tabela 3. Resultados Constructor Percepção de Facilidade de Uso

\begin{tabular}{|l|l|l|l|l|l|}
\hline & RM & Mediana & Não concordo (1-2) & Neutro (3) & Concordo (4-5) \\
\hline PFU1 & 4,46 & 4 & $0-0 \%$ & $0-0 \%$ & $13-100 \%$ \\
\hline PFU2 & 4,36 & 5 & $0-0 \%$ & $1-8 \%$ & $12-92 \%$ \\
\hline PFU3 & 4,62 & 5 & $0-0 \%$ & $2-15 \%$ & $11-85 \%$ \\
\hline PFU4 & 4,77 & 5 & $0-0 \%$ & $0-0 \%$ & $13-100 \%$ \\
\hline PFU5 & 4,15 & 4 & $0-0 \%$ & $3-23 \%$ & $10-77 \%$ \\
\hline
\end{tabular}

A análise dos dados coletados permitiu considerar que a hipótese de pesquisa do modelo professor, em que H0 se propunha avaliar se as áreas de atuação e experiências anteriores influenciavam no uso do modelo, não pode ser totalmente confirmada, pois mesmo entre os respondentes que afirmaram não ter experiências com jogos e tecnologias houve aceitação do modelo. No entanto, o resultado pode ser considerado relevante uma vez que a relação e a possibilidade de integração entre teoria e prática pode ter afetado positivamente as repostas e consequentemente a intenção de uso do modelo.

Quanto a hipótese H1 de verificar se a percepção de facilidade de uso tem impacto na percepção de utilidade, os resultados apontam fortes indícios de que seja verdadeira, uma vez que os resultados obtidos nesses itens apontam valores de mediana de 4,5 quando o indicado como aceitável, seria em torno de 4,0. Dessa forma, os resultados de $\mathrm{H} 0$ e $\mathrm{H} 1$ colaboram com a aceitação da hipótese $\mathrm{H} 2$ de que o modelo permite o uso em diferentes áreas de aprendizagem. Nesse sentido, é possível perceber pelas avaliações, que o Uchallenge pode auxiliar nesse processo, uma vez que o professor pode definir seus objetivos pedagógicos e decidir quais aspectos educacionais quer dar maior ou menor ênfase, sem, no entanto, limitar as opções dos alunos.

\subsection{Avaliação por alunos}

Não existe um consenso ao avaliar o uso de tecnologias para educação. Autores como Gladcheff et al. [2001] propõem uma metodologia, baseada em aspectos pedagógicos, com objetivos educacionais específicos, conteúdos e desafios. Savi [2011] propõe um modelo baseado na motivação dos alunos, experiência de uso e aprendizagem. O modelo apresenta ainda, um ambiente ubíquo, cuja natureza se integra ao ambiente real com interação através de sensores, produzindo dados personalizados para os usuários. 
VIII Congresso Brasileiro de Informática na Educação (CBIE 2019)

Anais do XXX Simpósio Brasileiro de Informática na Educação (SBIE 2019)

Segundo Nelly et al. [2008], pode-se considerar para avaliação desse tipo de tecnologia aspectos da interação como desempenho e impacto proporcionados pelo ambiente.

Os estímulos provocados pelo jogo podem produzir emoções e divertimento, o que aumenta as possibilidades de aprendizagem, uma vez que essas emoções produzem motivação que está estreitamente ligada a repetir sensações ou situações prazerosas. Dessa forma, é importante avaliar o grau de motivação gerado no aluno durante o jogo. Para isso, Keller [2009] sugere o uso do Attention, Relevance, Confidence, and Satisfaction (ARCS), que prevê quatro categorias importantes na motivação do aluno para aprendizagem (atenção, relevância, confiança e satisfação).

A atenção tem como objetivo ganhar o interesse dos alunos e mantê-lo durante a realização do jogo. A relevância indica se o estudante percebe que o jogo é uma necessidade. A confiança mostra se o aluno espera ter sucesso, e a satisfação envolve as possíveis recompensas que ele espera ao realizar a atividade. Dessa forma, considerando as diferentes possibilidades de avaliação de jogos educativos foi definido como base para a avaliação, uma adaptação do modelo de Savi [2011], que possibilita a análise da motivação baseando no modelo ARCS e Nelly et al. [2008] que prevê a análise de integração do aluno com o ambiente, conforme Tabela 4.

Tabela 4. Variáveis da avaliação do aluno

\begin{tabular}{|l|l|}
\hline \multicolumn{2}{|l|}{ Atenção } \\
\hline A1 & A interface do jogo é atraente. \\
\hline A2 & Os problemas e desafios propostos capturaram minha atenção. \\
\hline A3 & A variação de atividades ajudou a me manter atento ao jogo. \\
\hline \multicolumn{2}{|c|}{ Relevância } \\
\hline R1 & O conteúdo do jogo é relevante aos meus interesses na disciplina. \\
\hline R2 & O funcionamento e o ambiente do jogo estão adequados ao meu jeito de aprender. \\
\hline R3 & O conteúdo do jogo faz relação com conhecimentos que eu já possuía \\
\hline \multicolumn{2}{|c|}{ Confiança } \\
\hline C1 & O jogo é fácil de entender e utilizar. \\
\hline C2 & Ao passar as etapas do jogo senti confiança de que estava aprendendo. \\
\hline \multicolumn{2}{|c|}{ Satisfação } \\
\hline S1 & Estou satisfeito pois tive oportunidade de praticar o que estava aprendendo. \\
\hline S2 & É por meu esforço pessoal que consigo avançar no jogo. \\
\hline \multicolumn{2}{|c|}{ Integração com ambiente } \\
\hline I1 & O jogo promove momentos de cooperação e/ou competição entre as pessoas que participam. \\
\hline I2 & Os elementos reais do jogo (sensores, contextos e práticas) foram importantes para evolução e \\
\hline & conclusão do jogo. \\
\hline I3 & Diverti-me com o jogo e não vi o tempo passar. \\
\hline
\end{tabular}

Para avaliação do modelo Uchallenge com o aluno, foi construído um jogo chamado "Redescobrindo a América", o qual foi criado por um professor de Geografia de uma escola pública. $\mathrm{O}$ jogo teve como cenário os ambientes na própria escola. $\mathrm{O}$ professor criou para esse jogo cinco níveis de dificuldades. As ações do jogo alternavam problemas de Geografia sobre o continente americano, os quais fazem parte do conteúdo da grade curricular do $8^{\circ}$ ano do ensino fundamental. O processo de criação dos problemas foi desenvolvido previamente pelo professor, assim como os tipos de ações em cada etapa do jogo.

A população da turma que participou do jogo era composta de 22 alunos, com idade entre 13 e 15 anos, que foram cadastrados previamente no sistema e realizaram 
VIII Congresso Brasileiro de Informática na Educação (CBIE 2019)

Anais do XXX Simpósio Brasileiro de Informática na Educação (SBIE 2019)

teste de acesso. Os alunos fizeram uso da aplicação mostrada na Figura 2 e em seguida, responderam o questionário de avaliação do jogo.

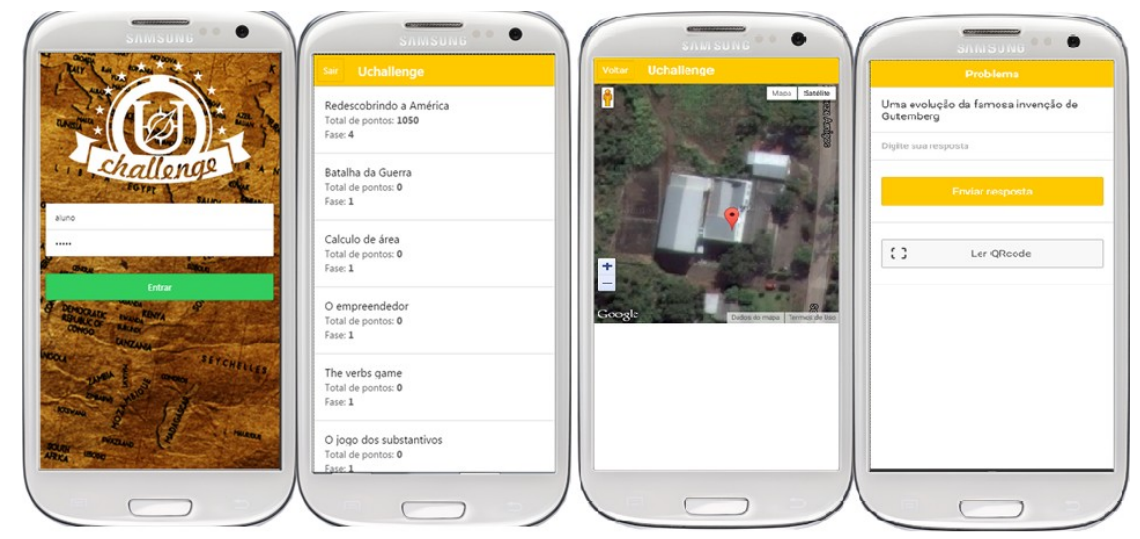

Figura 2: Protótipo usado para avaliação pelos alunos

Uma análise inicial dos dados, conforme mostra a Figura 3, permite-nos verificar a frequência das respostas obtidas em cada uma das variáveis. Esse tipo de análise, permitiu identificar os principais pontos positivos e negativos em cada item, identificando aspectos que podem ser melhorados, além de indicarem a homogeneidade ou heterogeneidade das respostas.

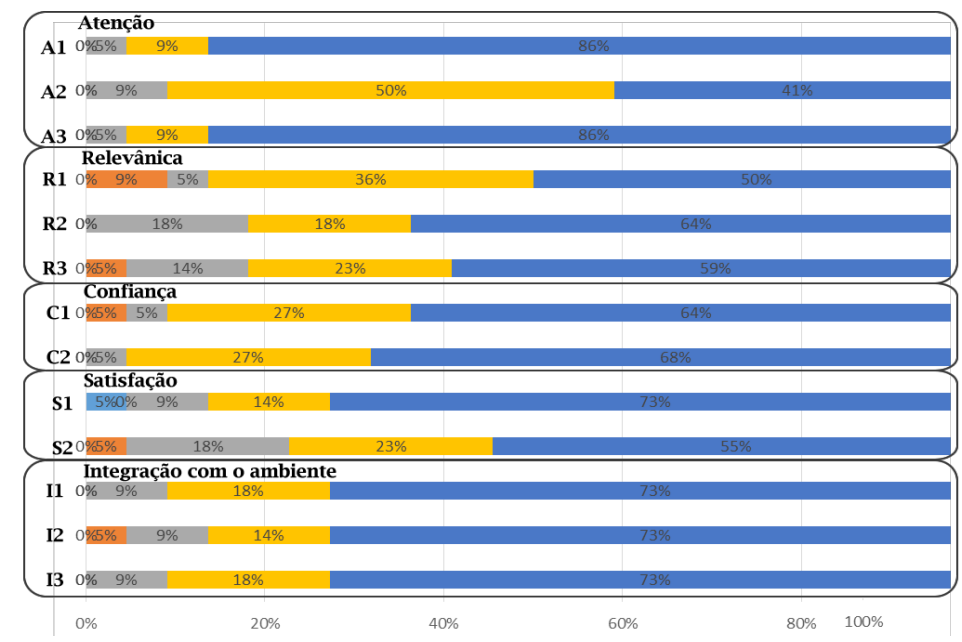

Figura 3. Frequência de respostas da avaliação de alunos

Pode-se perceber, dessa forma, que em praticamente todos os itens avaliados a soma dos itens 4 - concordo, e 5 - concordo totalmente supera os $80 \%$ de concordância. Dos itens do modelo ARCS, o que apresentou menor avaliação de concordância, foi o item "Relevância" em que os alunos avaliaram a relação do jogo com a disciplina. Os itens 2 - não concordo e item 3 de neutralidade tiveram uma variação de 14\% a 19\%.

Colaborando com os dados de frequência, foram analisadas médias e medianas de todos os itens, pode-se constatar que os níveis críticos desses valores ficaram entre 4,27 a 4,81 para média e 4 a 5 para mediana. A análise apresenta um cenário positivo para o uso dos jogos, considerando a motivação, a partir dos elementos do modelo ARCS, além disso, os itens que avaliaram a integração do jogo com o ambiente e sensores, obtiveram aprovação superior a $90 \%$. 
VIII Congresso Brasileiro de Informática na Educação (CBIE 2019)

Anais do XXX Simpósio Brasileiro de Informática na Educação (SBIE 2019)

Com base nos resultados obtidos com a experimentação realizadas pelos alunos, em relação à motivação gerada pelo uso do jogo e a percepção de integração do ambiente, observou-se boa aceitação do modelo, considerando, dessa forma, que os resultados são encorajadores e que poderiam ser ampliados para uma amostragem mais significativa, em outros ambientes e áreas de avaliação.

\section{Conclusão}

Esse artigo apresentou o modelo Uchallenge para construção de jogos sérios e ubíquos focado em uma metodologia de aprendizagem baseada em problemas. A partir do protótipo foram realizadas duas avaliações. A primeira envolveu professores, em que foi utilizada a metodologia TAM para avaliar a intenção de uso do modelo. A segunda foi realizada com alunos, usando o modelo ARCS, para avaliar a motivação e o engajamento proporcionados pelo jogo, bem como a integração do jogo ao mundo real.

Os resultados da avaliação foram significativos, 100\% dos professores indicaram intenção de uso do Uchallenge, vislumbrando a utilização de forma interdisciplinar e de apoio para diferentes práticas pedagógicas. Os dados coletados junto aos alunos apontam forte interesse dos alunos pelo jogo, demonstrando motivação e engajamento na atividade proposta.

Como sugestão de trabalhos futuros está a realização de avaliações com um número maior de alunos e professores, a fim de se identificar quais são áreas mais favorecidas pelo modelo, além de ampliar o diagnostico identificando as trajetórias de aprendizagem percorridas durante os jogos.

\section{Agradecimentos}

Os autores agradecem ao Conselho Nacional de Desenvolvimento Científico e Tecnológico (CNPq), à Universidade do Vale do Rio dos Sinos (Unisinos) e reconhecem especialmente o apoio do Programa de Pós-Graduação em Computação Aplicada (PPGCA) da Unisinos.

\section{Referências}

Cárdenas-Robledo, L. A., Peña-Ayala, A. (2018). Ubiquitous learning: A systematic review. Telematics and Informatics, 35(5):1097-1132.

Gladcheff, A. P., Zuffi, E. M., \& Silva, D. D. (2001). Um instrumento para avaliação da qualidade de softwares educacionais de matemática para o ensino fundamental. In Anais do XXI Congresso da Sociedade Brasileira de Computação.

Hainey, T., Connolly, T. M., Boyle, E. A., Wilson, A., \& Razak, A. (2016). A systematic literature review of games-based learning empirical evidence in primary education. Computers \& Education, 102, 202-223.

Huang, Y. M., \& Chiu, P. S. (2015). The effectiveness of a meaningful learning-based evaluation model for context-aware mobile learning. British Journal of Educational Technology, 46(2), 437-447.

Huizinga, J. (2008). Home ludens: o jogo como elemento de cultura. São Paulo. Perspectiva. 
VIII Congresso Brasileiro de Informática na Educação (CBIE 2019)

Anais do XXX Simpósio Brasileiro de Informática na Educação (SBIE 2019)

Hwang, G. J., Wu, P. H., Chen, C. C., \& Tu, N. T. (2016). Effects of an augmented reality-based educational game on students' learning achievements and attitudes in real-world observations. Interactive Learning Environments, 24(8), 1895-1906.

Keller, J. M. (2009). Motivational design for learning and performance: The ARCS model approach. Springer Science \& Business Media.

Khan, A. I., Al-Shihi, H., Al-Khanjari, Z. A., \& Sarrab, M. (2015). Mobile Learning (M-Learning) adoption in the Middle East: Lessons learned from the educationally advanced countries. Telematics and Informatics, 32(4), 909-920.

Klopfer, E., Sheldon, J., Perry, J., \& Chen, V. H. (2012). Ubiquitous games for learning (UbiqGames): Weatherlings, a worked example. Journal of Computer Assisted Learning, 28(5), 465-476.

Laine, T. H., Sedano, C. A., Joy, M., \& Sutinen, E. (2010). Critical factors for technology integration in game-based pervasive learning spaces. IEEE Transactions on Learning Technologies, 3(4), 294-306. LÉVY, PIERRE. Cibercultura. São Paulo: Ed. 34, 1999

Neely, S., Stevenson, G., Kray, C., Mulder, I., Connelly, K., \& Siek, K. A. (2008). Evaluating pervasive and ubiquitous systems. IEEE Pervasive Computing, 7(3), 8588.

Persico, D., Manca, S., \& Pozzi, F. (2014). Adapting the technology acceptance model to evaluate the innovative potential of e-learning systems. Computers in Human Behavior, 30, 614-622.

Prensky, M. R. (2012). From digital natives to digital wisdom: Hopeful essays for 21st century learning. Corwin Press.

Prieto, J. C. S., Migueláñez, S. O., \& García-Peñalvo, F. J. (2014). Mobile learning adoption from informal into formal: an extended TAM model to measure mobile acceptance among teachers. In Proceedings of the Second International Conference on Technological Ecosystems for Enhancing Multiculturality (pp. 595-602). ACM.

Santaella, L. (2014). Comunicação ubíqua: repercussões na cultura e na educação. Pia Sociedade de São Paulo-Editora Paulus.

Savi, R., Wangenheim, C., \& Borgatto, A. (2011). Um modelo de avaliação de jogos educacionais na engenharia de software. Anais do XXV Simpósio Brasileiro de Engenharia de Software (SBES), São Paulo.

Wallace, L. G., \& Sheetz, S. D. (2014). The adoption of software measures: A technology acceptance model (TAM) perspective. Information \& Management, 51(2), 249-259.

Weiser, M. (1991). The Computer for the 21 st Century. Scientific american, 265(3), 94-105. 